\title{
Costacutia n. nom. für die Gattung Acuticosta Grimm 1998 (Buchiolinae, Cardiolidae, Arcoida) aus dem Devon des Rheinischen Schiefergebirges (Deutschland)
}

\author{
Costacutia, new name for the genus Acuticosta GRIMM 1998 (Buchiolinae, Cardiolidae, Arcoida) \\ from the Devonian of the Rhenish Slate Mountains (Germany) \\ MATTHIAS C. GRIMM, Mainz
}

\begin{abstract}
Durch GRIMM (1998) wurde für die vorher unter der Gattung Buchiola BARRANDE 1881 zusammengefaßten devonischen Muscheln die neue Unterfamilie Buchiolinae (Cardiolidae, Arcoida, Lamellibranchiata) eingeführt. Die Unterfamilie besteht aus den Gattungen Buchiola BARRANDE 1881, Acuticosta GRIMM 1998, Glyptohallicardia GRIMM 1998, Planocardia GRIMM 1998, Levibuchiola GRIMM 1998 und Porsguenia GRIMM 1998. Der Name Acuticosta GRIMM 1998 ist jedoch nicht gültig, da eine primäre Homonymie zu Acuticosta SimPSON 1900 (Acuticostinae, Unionidae, Unionida) besteht. Die Gattung wird daher mit dem Namen Costacutia n. nom. benannt. Der neue Name ist ein Anagramm von Acuticosta. Einzige Art und Typusart der Gattung ist Costacutia acuticosta (SANDBERGER \& SANDBERGER 1854) [Cardiola retrostriatum $\mathrm{v}$. BuCH sp. var. acuticosta ]. Sie wurde von Grimm (1998: 54 f., Taf. 1, Fig. 7-9; Taf. 6, Fig. 4-7) unter dem ungültigen Namen Acuticosta acuticosta beschrieben und abgebildet.
\end{abstract}

\section{Dank}

Ich danke Herrn PhILIPPE Bouchet, Muséum National d'Histoire Naturelle (Paris), für den Hinweis zur Homonymie der Gattung.

\section{Literatur}

Grimm, M. C. 1998. Systematik und Paläoökologie der Buchiolinae nov. subfam. (Cardiolidae, Arcoida, Lamellibranchiata, Devon). - Schweizerische Paläontologische Abhandlungen 118: 1-135, Basel.

SANDBERGER, G. \& SANDBERGER, F. 1854. Die Versteinerungen des rheinischen Schichtensystems in Nassau - Mit einer kurzgefaßten Geognosie dieses Gebietes und mit steter Berücksichtigung analoger Schichten in anderen Ländern. Lieferung 6: 169-200, Taf. 24-28, Wiesbaden (Kreidel \& Niedner).

SiMPSON, C.T. 1900. Synopsis of the najades or pearly freshwater mussels. - Proceedings of the United States National Museum 22: 501-1044, Washington D.C.

Eingang des Manuskriptes am 10. November 1999; Annahme durch die Schriftleitung am 20. September 2000.

Anschrift des Autors: Dr. MatTHIAS Grimm, Eduard-Franck-Straße 12, D-55122 Mainz; e-mail: Kgrimm@mail.uni-mainz.de 